Revue européenne des sciences sociales

European Journal of Social Sciences

XXXVIII-117| 2000

Métaphores et analogies. Schèmes argumentatifs des sciences sociales

\title{
Déplier le rêve
}

Sabine Prokhoris

\section{(2) OpenEdition}

1 Journals

Édition électronique

URL : http://journals.openedition.org/ress/709

DOI : $10.4000 /$ ress.709

ISSN : 1663-4446

Éditeur

Librairie Droz

\section{Édition imprimée}

Date de publication : 1 février 2000

Pagination : 49-68

ISBN : 2-600-00409-2

ISSN : 0048-8046

Référence électronique

Sabine Prokhoris, "Déplier le rêve », Revue européenne des sciences sociales [En ligne],

XXXVIII-117 | 2000, mis en ligne le 17 décembre 2009, consulté le 13 mai 2019. URL: http://

journals.openedition.org/ress/709; DOI : 10.4000/ress.709 


\title{
Sabine PROKHORIS
}

\section{DÉPLIER LE RÊVE}

Que le premier langage dut être figuré.

\author{
Jean-Jacques Rousseau
}

Le langage est un labyrinthe de chemins. Vous venez par un côté et vous vous y reconnaissez; vous venez au même endroit par un autre côté et vous ne connaissez plus votre chemin.

Ludwig Wittgenstein

Un art des passages: ainsi pourrions-nous définir la psychanalyse, considérée dans son exercice d'abord, dans les mouvements par lesquels elle tente de penser, voire de légitimer cet exercice, ensuite, mouvements paradoxaux, comme j'avais tenté de l'indiquer ici même l'an dernier'.

Que signifie le parti d'une telle définition? Sur quoi prend-il appui? À quoi conduit-il si on le soutient dans toute sa rigueur, s'agissant de l'«éthique» de la psychanalyse, et partant de la problématisation de l'humain contemporain que l'invention freudienne inaugure?

Je tâcherai de préciser ces différents points, ayant en tête l'apologue du Minotaure (version Dürrenmatt) ${ }^{2}$ proposé à notre méditation, ainsi que les questions soulevées par l'argument de notre rencontre de cette année. Questions qui nous invitent à réfléchir autour de la question de la métaphore, en l'associant en quelque sorte à celle de l'espace. Association libre peut-être mais non dénuée de sens, d'autant qu'elle se trouve surdéterminée par la forme dédoublée qu'a voulu prendre, cette année, l'argument offert à notre méditation: quelques pistes, conceptuellement formulées, au sujet de la métaphore, et un conte du Minotaure et de Thésée en leur labyrinthe. Dédoublement non sans effets immédiats: car si la métaphore est affaire de passages, le labyrinthe où se terre le malheureux Minotaure n'est-il pas justement un dispositif de passages à tout coup empêchés? En ce sens, l'anti-métaphore même, dans cette figure de détours cauchemardesquement voués à l'échec, sauf à suivre un seul itinéraire? Détours prescrits, dans une cage. Or écrivant ces lignes, je m'aperçois que j'ai en tête une séquence du Satyricon de Fellini, la séquence du labyrinthe justement, incroyable, subversive, car le laby-

Troubles, in «Revue européenne des sciences sociales», t. XXXVII, 1999, Nº 113, pp. 45 ss. Friedrich Dürrenmatt, La mort de la Pythie, suivi de Minotaure, Lausanne, L'Age d'Homme, 1990. Ce récit a servi de référence pendant les travaux du colloque; on en trouvera un résumé dans la contribution introductive de Marie-Jeanne Borel, dans le présent numéro de la Revue. 
rinthe de Fellini n'est pas un souterrain : il est à ciel ouvert, et c'est ainsi que nous sommes invités à le voir. Et cette perception, dans la dimension d'une étendue sans cesse barrée, nous fera mesurer la violence et l'arbitraire contenus dans l'architecture de dédales, dans l'ingénieuse construction de l'empêchement des passages. Où nous serait donnée à voir le plus psychanalytiquement possible la question de l'impasse: nous n'oublierons pas ici, et tout au long de la présente réflexion, que celui qui s'adresse au psychanalyste le fait parce qu'il est acculé, parce qu'il se cogne la tête contre les murs. S'agira-t-il alors de trouver comment parcourir les couloirs dans le sens ordonné par le labyrinthe, et donc, si l'on peut dire, de marcher droit (droit sur le Minotaure pour lui régler son compte, puis droit vers la sortie), muni du fil d'Ariane, ou de pouvoir déconstruire le labyrinthe, dissoudre les lignes qui en dressent le plan, au risque évidemment de laisser le Minotaure gambader en tous sens? Mon choix de psychanalyste, je l'indique très clairement ici, pour ensuite m'en expliquer, sera de combattre plutôt le labyrinthe qu'un hypothétique Minotaure, avec, pour arme étrange, ou instrument, non pas un fil (et d'ailleurs, le destin de Thésée, pas plus que celui d'Ariane ne sont pour me tenter), mais l'écheveau échevelé de la métaphoricité. En guise de viatique pour accompagner la divagation, non sans buts cependant, que j'ai envie d'entreprendre, je vous proposerai, de surcroît, ce mot de Michel Foucault: «C'est le labyrinthe qui fait le Minotaure, non l'inverse.» ${ }^{3}$

Quoi qu'il en soit, l'idée même de passage, que j'utilise pour d'entrée de jeu cerner et discerner la question de la psychanalyse, porte et traverse, si l'on peut dire, tant l'affaire de la métaphore que celle de l'espace, quelle que soit la dimension de réalité que ce déplacement affecte et ainsi modifie, donc «fictionne »: fait être autrement. Et j'assortirai cette définition initiale de l'art de la psychanalyse la psychanalyse qui, faut-il le rappeler, est une méthode de traitement de la souffrance psychique - de la thèse suivante: le psychique est un espace que structure, y compris dans sa temporalité, «si peu chronologique», ainsi que nous le font percevoir les étranges jeux de la mémoire et de l'oubli, une métaphoricité originaire, c'est-à-dire une relation au sens marquée par de l'absence, par de l'écart, par de l'inadéquation, entre des éléments qui nous prennent, nous orientent et tout autant nous égarent, dans les leurres des ressemblances les plus ténues par lesquelles nous nous efforçons de les relier à nous. Ramener l'inconnu à du connu, en nous figurant que, par l'un de ses bouts au moins, il pourrait offrir quelque ressemblance avec lui, il pourrait être reconnu. Rêver l'inconnu, donner forme, au moyen de cet enveloppement par l'espèce de matière imaginaire que nous secrétons, à ce rien de sens sur quoi il nous fait buter. S'agissant de la souffrance psychique et de son traitement, je dirai que cette souffrance aurait trait à une sorte de rigidification et du coup de repli, par sclérose et dessèchement, des circuits métaphoriques, alors soudain alourdis, saturés d'un sens coincé, dont l'arbitraire abusif, massif, paralyse alors tout mouvement. Arrêt sur image. Sens de plomb. Et nous voilà avec un beau labyrinthe, lequel n'est peut-être rien d'autre qu'un rêve replié, archi raidi d'empois.

Comme vous vous en apercevez, j'ai envie, pour naviguer dans les courants de la problématique vaste et plutôt mouvante qui s'étend devant nous, en somme de

Michel Foucault, Dits et Écrits, t. I, p. 424. 
profiter de la barque que nous lance cette conjonction un brin inattendue d'une réflexion proprement théorique et d'un texte littéraire, car le décalage qu'elle fait jouer du conceptuel au poétique, en ce que ce décalage initie comme impulsion à penser, en la manière surtout dont il fait procéder cette impulsion, me semble non seulement fécond, mais quasiment mettre en scène une des dimensions, et non la moindre, de la problématisation que nous avons à mener. À prendre ainsi au mot - et non à la lettre - le jeu qui nous est proposé, il est clair que dès le départ, je n'envisage nullement la nouvelle de Dürrenmatt comme une illustration venant adoucir la potion rébarbative d'un discours sèchement conceptuel, pour mieux la faire passer; pas davantage comme le niveau préalable à une «vraie» pensée articulée ne pouvant quant à elle ne nous être délivrée qu'une fois notre esprit, que certes l'œuvre d'imagination aura pu éveiller, affranchi de ses enfantines séductions: où l'œuvre artistique se verrait réduite au rang d'une simple métaphore pédagogique, à ne surtout pas laisser déborder en tout sens, ou faire rêver, métaphore tout entière orientée vers une vérité conceptuelle pressée de la laisser choir derrière elle comme une vieille mue vouée à se dessécher. En fait, je considérerai l'écart entre la nouvelle de Dürrenmatt et le texte de Marie-Jeanne Borel comme comme une mise en tension de la question même de la métaphore dans la pensée. S'ouvre alors un espace de réflexion configuré de telle sorte que nous puissions nous déprendre de certaines habitudes interprétatives, de certaines hiérarchies discursives propres à écraser la question de la métaphore sous la pesanteur de la vocation qu'on lui prête à signifier, fût-ce imparfaitement, et dans une sorte de danse des sept voiles, une vérité mieux dite hors de ses chatoiements à surveiller sans cesse. C'est-à-dire que si, dans une sorte d'attention également flottante de notre lecture - et je ne me priverai pas en l'occurrence d'user d'un de mes instruments intellectuels de psychanalyste, que je vous propose d'emprunter pour me suivre - nous considérons ces deux textes à égalité de statut, sur des plans homologues, leur décalage même autorisera alors des passages, des continuités de l'un à l'autre de ces plans, à la faveur desquels un certain nombre de propositions concernant la question de la métaphore, ou, aimerais-je mieux dire, du mouvement métaphorique, pourront être énoncées. Je ferai donc jouer ces deux textes, à la teneur fort éloignée mais aux effets conjoints, puisqu'aussi bien ils nous arrivent ensemble pour tisser le canevas de notre rencontre de cette année, l'un avec l'autre, mais quasiment en leur tournant le dos - en m'adossant à eux, pour parler plus poliment - ce qui veut dire en ne cherchant ni à être fidèle à leur message supposé (pour ce qui est du texte de Dürrenmatt en tout cas), ni à les interpréter l'un par l'autre, en un cercle herméneutique qui pourrait bien n'être en l'occurence rien de plus qu'un cercle vicieux. Quel que soit donc l'usage, partiel, que je pourrai faire des significations qu'ils élaborent chacun à leur manière, mon propos s'inscrira d'abord dans l'orbe de certains effets nés de leur conjonction, nés, avanceraije, de l'étonnante figure de redoublement dont ils affectent notre lecture et, partant, le mouvement de pensée, qu'ils ont pour fonction, telle est la règle de l'exercice qui nous réunit, d'initier. Ensemble et décalés, et par le décalage même qui les tresse l'un à l'autre, ils portent et tissent l'affaire de la métaphore et de ses enjeux. Ma méthode se montrera finalement assez docile aux termes de la donne proposés par nos hôtes; elle me permettra en tout cas d'emblée de formuler ceci, comme résumé-traduction de la question que je me propose de traiter chemin faisant: l'espace de la métaphore: de quoi y retourne-t-il? Ou encore pour faire un 
pas de plus: la dimension que l'on peut dire poétique de la pensée - et de l'existence - quelle matérialité vise-t-elle à accomplir? Et selon quelles procédures? Questions que j'assortirai immédiatement d'une première proposition, laquelle j'essaierai de rendre aussi claire que possible: non seulement la métaphore fonctionnera comme un certain espace, mais encore cet espace est un espace originaire. Par ailleurs, cet espace est aussi un espace non clos, puisqu'une métaphore peut toujours ouvrir sur d'autres, en amont, en aval, à côté, et que son fonctionnement ne souffre par conséquent pas de bords externes, de frontières chargées d'en délimiter le territoire (quoi qu'en ait, un peu dépité, un certain régime philosophique à ce sujet, comme nous l'a fait voir dans sa contribution Jean-Jacques Wunenburger); il se déploie en même temps, cependant, comme un espace non homogène, parcouru, comme par des vagues, de bords «internes» si l'on peut dire, c'est-à-dire de sortes de fêlures constitutives, - ces lignes de vide dont nous a parlé Jean-Claude Passeron citant Tchouang tseu. Quant au caractère originaire, c'est-à-dire en somme premier quant à sa fonction, que je lui prête, pour la pensée et l'existence, c'est en un sens très paradoxal que nous le verrons jouer. Ainsi serat-il, cet espace de la métaphore, a priori, au sens strict, mais seulement aprèscoup, c'est-à-dire en un temps second.

J'emploie ici ce terme d' «après-coup», dont Proust nous a fait très précisément voir quelle expérience de présence véritable il décrit, de présence sur fond d'absence et d'initial ratage du lien au monde, au sens rigoureux où la psychanalyse l'a fait travailler comme un concept. Mais partons de Proust. Voici par exemple un des nombreux passages de la Recherche qui nous décrit cela:

\begin{abstract}
... je m'essuyai la bouche avec la serviette qu'il m'avait donnée; mais aussitôt, comme le personnage des «Mille et Une Nuits » qui sans le savoir accomplissait précisément le rite qui faisait apparaître, visible pour lui seul, un docile génie prêt à le transporter au loin, une nouvelle vision d'azur passa devant mes yeux; mais il était pur et salin, il se gonfla en mamelles bleuâtres; l'impression fut si forte que le moment que je vivais me sembla être le moment actuel; plus hébété que le jour où je me demandais si j'allais vraiment être accueilli par la princesse de Guermantes ou si tout n'allait pas s'effondrer, je croyais que le domestique venait d'ouvrir la fenêtre sur la plage et que tout m'invitait à descendre me promener le long de la digue à marée haute; la serviette que j'avais prise pour m'essuyer la bouche avait précisément le genre de raideur et d'empesé de celle avec laquelle j'avais eu tant de peine à me sécher devant la fenêtre, le premier jour de mon arrivée à Balbec, et maintenant, devant cette bibliothèque de l'hôtel de Guermantes, elle déployait, réparti dans ses pans et dans ses cassures, le plumage d'un océan vert et bleu comme la queue d'un paon. Et je ne jouissais pas que de ces couleurs, mais de tout un instant de ma vie qui les soulevait, qui avait été sans doute aspiration vers elles, dont quelque sentiment de fatigue ou de tristesse m'avait empêché de jouir à Balbec ${ }^{4}$.
\end{abstract}

Citation un peu longue, mais qui permet de saisir comment la présence pleine, parcourue cependant d'une fondamentale cassure qui seule la rend possible - et ce signifiant de «cassure», comme mot et comme chose ou comme expérience, est au cœur de cette page -, comment cette présence animée par la puissance d'un désir ignoré qu'éveille soudainement une logique métaphorique, sera donnée pour de vrai après coup, dans le redoublement et la métamorphose d’un écart: la pre-

4 Marcel Proust, À la recherche du temps perdu, Paris, Gallimard, 1973, Bibliothèque de la Pléiade, t. III, pp. $868 / 9$. 
mière fois, le narrateur fut absent à sa perception: premier écart. La deuxième fois, par le miracle imprévu d'une ressemblance aussi intense qu'infime entre une sensation actuelle et une sensation passée quant à elle reliée à l'absence initiale du narrateur à ce qu'il vivait alors, cette deuxième fois qui sera l'instant où la perception se donne et se matérialise véritablement dans l'espace psychique qui l'accueille et la désire, adviendra en l'absence de ce qui ne fut pas vécu bien qu'ayant été présent. Un vide se creuse, au sein duquel se met à exister pour de bon in absentia, ce dont il deviendra enfin, et par hasard, possible de jouir: second écart. Écart d'un après-coup, puisque la première expérience digne de ce nom a lieu en un temps second. On notera bien sûr qu'elle survient par un chemin très exactement métaphorique. Et c'est le lien entre ces deux temps et ces deux formes d'absence - absence du narrateur à son «présent» à un moment $t$, absence de l'objet perçu à un moment t' - structure de la métaphore et son œuvre, qui rémunère, pour reprendre, à propos de l'existence, le mot de Mallarmé sur la poésie, le défaut, l'infirmité native, de notre lien au monde.

Emprunter ici un chemin tracé par Proust, avant de préciser schématiquement le sens psychanalytique de cette expression: l' «après coup», me permet également d'expliciter ce que je désignerais comme la vérité matérielle, pour reprendre encore une expression freudienne, de la poéticité dans une existence humaine. La vérité matérielle, c'est très précisément ce cadeau de présence profuse que la métaphoricité, au sens où nous venons d'en avoir la description dans la page de Proust, offrira aux insuffisants que nous sommes. Dimension qui porte un enjeu thérapeutique au sens le plus fort. Un enjeu politique également, foncièrement, pour des raisons qui s'élucideront un peu plus tard dans mon propos, dont je peux déjà dire qu'elles concernent le destin des relations d'emprise. Et là nous retrouvons l'affaire du labyrinthe et de ses pièges. Mais de l'enjeu thérapeutique, les liens avec l'autre dimension, la politique, se dessineront d'eux-mêmes, pour autant qu'il concerne le possible déverrouillage des relations d'emprise. On peut y réfléchir en tout cas, avant de reprendre directement la question de la psychanalyse, au moyen de la méditation foucaldienne sur la folie comme absence d'œuvre, et sur l'œuvre comme non-folie ${ }^{5}$, c'est-à-dire comme ce qui résiste aux assignations et aux partages qui contraindraient à la folie. Assignations dont un certain régime du discours de la psychanalyse, cependant, prompt à brandir la Loi et à sacraliser ses théories, peut se faire l'instrument. Un certain régime aussi de son exercice, que j'interrogerai un peu plus loin. Si l'œuvre, en tout état de cause, trouve dans l'art un espace d'expérimentation privilégié, c'est tout autant son expansion dans l'existence même que j'ai, comme psychanalyste en tout cas, en vue. L'œuvre non comme but formel, mais, selon le mot de Foucault, comme «relation à l'innommé » ${ }^{6}-$ lequel n'est pas l'«innommable»-, surgie de l'élan déployé pour donner un espace à ce qui n'est pas encore dans nos mots, pas encore dans nos vies. Au bout du compte, cela qui permettrait que nos existences se vécussent comme le rêve continué par d'autres moyens. Moyens à inventer, hors du solipsisme, moyens susceptibles de questionner, de violenter parfois, le laby-

Voir Michel Foucault, La folie, l'absence d' cuvre in L'histoire de la folie à l'âge classique, Gallimard, 1972, p. 575; et Dits et Écrits, tome I, pp. 172-188.

$6 \quad$ Michel Foucault, Dits et Écrits, t. 4, p. 256. 
rinthe des normes et conventions qui nous emprisonnent et nous «minoterrorisent ». Je soutiendrai pour ma part que la tâche du psychanalyste se situe très exactement en cette zone. Là également son éventuel refus de cette tâche, refus lourd de conséquences, ainsi que l'abus qui s'ensuit, issu d'une certaine propension à perfectionner le labyrinthe. Nous verrons un peu plus loin comment, et au nom de quoi. En attendant, Proust encore:

\begin{abstract}
La grandeur de l'art véritable, au contraire de celui que monsieur de Norpois eût appelé un jeu de dilettante, c'était de retrouver, de ressaisir, de nous faire connaître cette réalité loin de laquelle nous vivons, de laquelle nous nous écartons de plus en plus au fur et à mesure que prend plus d'épaisseur et d'imperméabilité la connaissance conventionnelle que nous lui substituons, cette réalité que nous risquerions fort de mourir sans avoir connue et qui est tout simplement notre vie $^{7}$.
\end{abstract}

Revenons à l'après-coup, tel que la psychanalyse en construit la notion. Cela nous permettra d'avancer un peu dans notre affaire du labyrinthe en son lien crispé à la question de la métaphore - en d'autres termes, dans la problématique de l'emprise et du sens, unique ou non. Car la théorisation psychanalytique de cette notion fera apparaître comment la dimension structurellement métaphorique de la temporalité psychique s'articule sur la question des enjeux les plus vitaux de la relation à l'autre, autour de ce que Freud décrit comme la détresse, la désorientation, l'espèce de prématuration fondamentale qui nous échoît en partage, et dont tout serait bon pour colmater l'angoisse. Parfois à n'importe quel prix, fût-ce au plus exhorbitant. Plus précisément, cela signifie que la relation première d'un petit humain au monde passe par l'intensité de sa dépendance à un autre, à un autre dont il est irréparablement séparé, et que le lien se noue dans toute sa force sur la rencontre, en cet autre qui se penche sur lui, de la cicatrice oubliée de cette épreuve initiale, qui s'éveillera, plus ou moins confusément, dans le contact avec la situation du nouveau venu au monde. Cicatrice recouverte par des constructions qui sont autant de mises en sens, fragiles et obstinées, de cette ligne de fêlure. Ces mises en sens constituent ce que Freud appellera le proton pseudos hystérique ${ }^{8}$, soit quelque chose que l'on pourrait décrire comme de la confiture étalée sur une absence de tartine: étrange situation qui, on le comprend, masque cette absence tout autant qu'elle la trahit: autrement dit du plein (de sens) à même du vide, décalant la perception de ce dernier. Opération qui pourrait être considérée, d'un certain point de vue, comme celle même de la métaphore.

Or cette structure de la relation du psychisme au monde est telle que c'est seulement dans un temps second, par un effet de ressemblance, plus ou moins ténu, plus ou moins arbitraire, entre deux moments, et parce que le momen $t a$ ' se trouve saisi dans un univers de significations, c'est-à-dire dans un univers tissé de langage - de relations signifiantes aux autres -, qu'un moment $a$ se met à exister, au sein de cet univers, qui l'y inscrit en en prescrivant la figure; et ladite prescription porte ici les deux sens du mot: ordonner - principalement à des fins supposées bénéfiques, voire thérapeutiques -, et décréter l'oubli en vue d'une amnistie. Et si l'on s'arrête un instant à cette dimension qui concerne, quant à elle, la Cité, l'on

Marcel Proust, À la recherche du temps perdu, op.cit., p. 895.

8 Sigmund Freud, Esquisse d'une psychologie scientifique, in La naissance de la psychanalyse, Paris, PUF, 1973, pp. 363-369. 
sait, Nicole Loraux l'a fort bien montré', que nulle amnistie n'est dénuée d'un but politique précis: à savoir que l'union dans la communauté puisse exister et perdurer. Pour en revenir à la considération des existences singulières, perspective au moyen de laquelle la psychanalyse saisit la question de l'humain, c'est à propos de ce qu'elle désigne comme traumatisme que cette dimension de l'après-coup va se trouver dégagée. Le traumatisme sera justement cette initiale fêlure constitutive du psychisme, qui advient dès lors que le sujet humain se trouve confronté à une situation d'intensité qu'il n'a, au moment où elle se produit, aucun moyen d'affronter, à laquelle il ne peut d'aucune façon donner un sens quelconque, et qui le laisse dans la déréliction, la démunition, l'obscurité les plus totales. La teneur et le degré de cette intensité seront fruit du choc de la rencontre de l'autre, du semblable, en ce qu'il a de plus énigmatique: à savoir en cette zone obscure, indistincte, d'absolue communauté dans l'épreuve de solitude et de dépendance mutuelle, parée des déguisements signifiants que l'adulte, pris en eux sans le savoir, imposera à l'infans, et par lesquels ce dernier se reliera au monde, à la faveur d'occasions lui permettant d'en revêtir le dénuement premier alors ainsi voilé. Si donc ce qui constitue pour un sujet l'insensé initial en événement, et en événement non seulement premier mais originaire - un originaire qui est une pure et très réelle fiction - s'effectue sous la modalité d'une mise en sens à partir d'un événement ultérieur pouvant avoir comme un air de famille avec le premier, cette logique de ressemblance se mettant à jouer dès lors que cet événement second se trouve pris dans un rêts de signifiants alors disponibles, qui viennent offrir au sujet de quoi se repérer (et censément se réparer), on comprend ce que nous soutenions plus haut: qu'un régime de part en part métaphorique constitue l'espace tout à la fois de la pensée et de l'existence. On comprend aussi en quel sens la métaphoricité peut être dite a priori dans sa structure d'après-coup même. La métaphoricité et ses incessants décalages, sources d'autant d'ombres que de lumière portée sur les choses, sorte de fondamentale défaite en même temps que de victoire sur l'écart d'un sujet au monde, et ainsi lien au monde humain par le biais des mises en sens, en figures, en tropes en somme, dont cet univers foisonnant de signifiants-écrans, fournit, plus ou moins confusément, les éléments. Et dans ce proton pseudos (premier mensonge, ou mensonge premier) hystérique, noyau constitutif du psychisme en son extimité, et j'expliciterai dans les lignes qui vont suivre ce néologisme qui n'est pas de moi, mais dont on devine, après ce que nous venons de décrire, ce qu'il tend à désigner, dans ce proton pseudos donc gît la source de toute capacité à rêver, mais tout autant la menace de labyrinthe, qui porte en elle la condamnation à rencontrer l'altérité comme monstrueuse. Une altérité qui n'est pourtant que l'image effrayante de l'altération dont nous sommes tissés dans notre relation à notre semblable, à notre prochain: le Minotaure, ce monstre, est, comme le faisait remarquer lors de l'une de nos discussions Hervé Le Bras, un être mixte; davantage, il est une figure traversée d'hétérogénéité. C'est cette insupportable image d'un être fait d'hétérogène qu'il faudra tuer. Mais que s'agira-t-il de mettre à mort, si ce n'est, dans le Minotaure, notre extimité même, c'est-à-dire cette matière fissurée, altérée dont nous sommes faits, cette présence de l'autre, vide et pleine en soi, mouvante, insupportablement ouverte peut-être? Ouverte comme

9 Voir Nicole Loraux, La cité divisée, Paris, Payot, 1997. 
l'Océan: d'où la nécessité dont s'assortit le meurtre du Minotaure de trouver quel est le sens, le sens unique de l'impitoyable labyrinthe. Ouverte comme l'Océan, dont le même Hervé Le Bras, citant un livre sur la Géométrie du labyrinthe, nous faisait remarquer qu'il était le contraire du labyrinthe, parce qu'on ne s'y rencontre pas. J'aurais tendance à dire que c'est plutôt qu'on peut y rencontrer, par hasard, n'importe qui, ce qui explique l'impérieux besoin et l'impatience qui poussent Achab, fort heureux sans doute s'il pouvait s'engouffrer dans un labyrinthe, à tomber sur Moby Dick: Minotaure inversé, dans sa pure blancheur de pure baleine. Quoi qu'il en soit, le Minotaure, quant à lui, n'est pas n'importe qui, puisqu'il est d'emblée identifié comme l'ennemi. Autant dire l'Autre avec un grand A. Ce qui lui confère un genre sûr. En ce qui concerne du reste cette identité de l'altérité de l'autre avec une figure identifiable comme étant celle de l'ennemi, un certain nombre de remarques freudiennes, dans L'esquisse d'une psychologie scientifique d'abord, puis dans les textes de la Métapsychologie sur la haine, enfin le texte fameux sur la Négation ${ }^{10}$ nous intéressent ici, car ils font naître l'«identité» de soi à soi d'un mouvement d'expulsion de tout ce qui pourrait faire ressentir sa propre présence au monde en proie au trouble ou à l'altération: quête de toutes façons vouée à l'échec que celle de pareille «identité » tranquille, car se débarrasser de la fêlure qui tout à la fois nous lie et nous sépare de l'autre, et à laquelle les plus intenses rapports à un autre aimé nous poussent à nous identifier d'un coup, cela est l'impossible même. Un impossible que questionne, vivement, toute rencontre amoureuse en son ambivalence. Ambivalence foncière en effet, d'où l'espèce de colère qui fréquemment s'empare de qui tombe amoureux, si l'amour est cette bizarre expérience d'écho entre des régimes singuliers de tropes, c'est-à-dire entre des façons de se rêver au monde, qui, par hasard, parfois seulement sur de menues brindilles d'existence, se découvrent comme extrêmement familières entre elles, et ainsi se reconnaissent. D'où du même coup l'élan pour s'en déshabiller, de ces tropes: la nudité à laquelle alors confronte l'amour est celle même que ces systèmes de tropes qui ont aimanté les amants n'auront eu de cesse d'oblitérer: comment alors supporter cela? On se souviendra ici que Rousseau, dans l'Essai sur l'origine des langues, texte sur l'origine hélas! métaphorique du langage, fit de l'amour, «né du pur cristal des fontaines» - ce miroir qu'un rien vient troubler - la source du parler. L'amour, dont Lacan écrivit que c'est «donner ce qu'on n'a pas à quelqu'un qui n'en veut pas », aphorisme qui, s'il n'a guère de sens psychologique, en a un métapsychologique, l'amour enfin à propos duquel Freud nota qu' «un solide égoïsme préserve de la maladie, mais [qu'] à la fin on doit se mettre à aimer pour ne pas tomber malade, et qu'on tombe malade lorsqu' on ne peut aimer par suite de frustration ${ }^{11}$. Rude condition. Si nous traduisons cette sorte d'abstraction métapsychologique, soutenue, en des termes différents, et par Freud et par Lacan - abstraction au sens d'une extrême condensation, quasi onirique, et d'une totale vitesse de la pensée - si nous la traduisons en termes qui prendraient davantage leur temps, cela revient tout simplement à dire que c'est à ce dont nous ne voulons surtout pas en nous, à cette incicatrisable expérience de l'indénouable mixte d'absolu besoin d'un autre pour tout

\footnotetext{
10 Sigmund Freud, in Résultats, idées, problèmes, t. II, p. 135.

11 Sigmund Freud, Pour introduire le narcissisme, in La vie sexuelle, Paris, PUF, 1977, p. 91.
} 
simplement survivre humainement et d'inadéquation radicale de cet autre, qui répond par les signifiants qui l'habitent, à effacer la séparation d'avec lui, c'est à cette expérience dont la cruauté affecte tout un chacun (un peu comme si nos mains nous étaient toujours et foncièrement étrangères, quand bien même nous ferions tout pour oublier cela), que nous nous identifions chez un autre que nous aimons. C'est cela aussi que nous jetons sur lui, pour nous en débarrasser sans doute, mais aussi comme un lasso. Si bien que l'aimer, ou accepter son amour, revient à réouvrir au noyau de nous-mêmes cette constitutive épreuve d'absence, dont nous sommes affectés, cette histoire de vide recouvert du chatoiement de l'univers signifiant. Certes, les destinées singulières nouent chacune à leur manière cette défectivité foncière, et le trouble amoureux, ainsi que l'espèce de désarroi ou de rage qu'il déclenche plus ou moins clairement chez qui s'y trouve pris, naissent de la rencontre, hasardeuse, chez un autre alors étrangement familier, quelles que soient la distance «réelle» qui m'en sépare, d'une configuration de ce nœud telle qu'elle éveillera la mémoire de cette haïssable présence en «soi » d'un autre non seulement défaillant, mais lui-même traversé de cette initiale fêlure: cela même qui le fait si proche et si inconnu tout à la fois, cela même qui serre si fortement le lien mutuel. Réminiscence haïssable donc - «il n'y a pas d'amour heureux », écrivait Aragon, même si - et parce que - l'amour peut rendre si heureux. Haïssable d'autant plus pour qui tendra de toutes ses forces à la constitution, à la fabrication d'un «quant à soi» sans traces de cette douleur, de cette désorientation. Épreuve de l'autre en soi, l'autre étrangement familier. Un autre lui-même de part en part altéré: le mixte même, dont est fait l' «extime». Dimension que l'on peut faire le choix soit d'accueillir, en lui laissant tout simplement de l'espace, soit d'expulser, comme étrangère, monstrueuse, soit encore, en une sorte de compromis méfiant, de tenter de domestiquer: et là s'ouvre un autre chapitre, dans lequel nous aurions à parler peut-être de figures telles que celle de Médée, l'étrangère, ou c'est alors la question de la relation aux femmes et au «féminin », telle qu'elle est construite dans nos discours et dans nos pratiques, qui surgit. Nous en dirons un mot un peu plus loin. Quoi qu'il en soit, ce que nous pouvons pour l'heure avancer, c'est que la «monstruosité» n'est qu'un effet de perception, et si convaincante et convaincue soit cette perception, elle ne procède de rien d'autre que du refus farouche, raidi, de cette «extimité», ainsi traduite et refoulée. Extimité dont la menace de retour, c'est-à-dire de simple présence, présence qui vient informer, configurer, l'espace propre, fait horreur.

Extimité, donc. C'est à un artiste, danseur chorégraphe, Alain Buffard, que je dois d'être amenée à réfléchir avec ce vocable. L'une de ses dernières créations s'intitule en effet Intime/Extime, et elle se déploie dans un espace qu'elle fait naître de la rencontre entre les singularités : quatre danseurs, en deux trios successifs voués chacun, sous des aspects différents, à explorer les passages possibles d'une altération de corps à une autre à travers les aléas des corps à corps les plus «intimes», et aussi en parcourant la géographie propre d'un corps inconnu à luimême, que le contact avec un autre corps, avec toute autre masse en fait pouvant représenter métonymiquement un corps ou un fragment de corps en son énigme, redessinera à neuf: autrement dit, ce qui apparaît, et c'est la force et l'intensité poétique de cette performance, dans la matérialité d'un espace chorégraphique extraordinairement libre, c'est l'étendue potentiellement infinie de chaque singularité, étendue toujours hétérogène à elle-même, traversée de mouvantes lignes 
qui sont autant de traces perpétuellement recomposées de l'autre en soi: des élans, des langages, des sensorialités, des vitesses, étrangères, qui font le «propre» non comme territoire enclos, mais comme incessante et fondatrice altération, ce qu'on a coutume d'appeler l' «intérieur», ou l'«intime», n'étant au bout du compte que de l' «extérieur» replié. Extravagance native du familier.

Or, et nous ferons là un pas de plus, cet «extérieur», qu'est-ce sinon la masse du langage - verbal bien sûr, mais point de mots sans l'espace des corps où ils sont pris -, «ce vaste instrument de communication », ce «tissu arachnéen de mes rapports avec les autres poussant de tous côtés ses antennes mystérieuses », selon les mots de Michel Leiris ${ }^{12}$, dont la réalité, nous dit-il aussi, dépasse chacun et lui est radicalement extérieure, tout en étant la texture même, vitale, du plus proche: «si près », dit-il, « de mes viscères »; ce qui s'éprouve dans l'acte même de donner de la voix. Dans la performance chorégraphique ainsi intitulée, d'ailleurs, l'on donne de la voix, et le langage articulé parcourt le corps de verbes qui disent, de la tête aux pieds, son extimité justement, soit sa situation de toujours et partout pouvoir être traversé par une érotique, au bord à chaque instant de se retourner en monstruosité, tout au moins, et c'est la part, généreusement et audacieusement donnée au spectateur (Alain Buffard préfèrera quant à lui dire au «témoin »: autre figure de l'extime: le témoin est, contrairement au spectateur, dans une position indistinctement de « dehors » et de «dedans »), dans la perception, tout à la fois très précisément guidée et ouvertement demandée comme aléatoire, que ce dernier offrira à cette performance. Une perception plus ou moins disposée à se laisser traverser par ce qui la sollicite sans concession, mais non sans un charme et une grâce par moments intensément ludiques. Une érotique, c'est-à-dire ce jeu de l'autre aux mouvantes frontières de soi, jusque dans les gestes les moins apparemment adressés ou ouverts.

Sans le savoir, Alain Buffard, inventant ce néologisme pour décrire ce que problématise avec force sa danse, retrouvait un terme imaginé autrefois par Lacan, commentant le Freud de L'esquisse et de L'inquiétante étrangeté dans le séminaire qu'il prononça sur l'Éthique de la psychanalyse. Le Freud qui décrit la constitution de ce proton pseudos à partir de l'expérience de détresse originaire du nourrisson livré aux signifiants dont l'adulte l'emmaillotera en quelque sorte, un adulte plus ou moins emmailloté lui-même, c'est-à-dire plus ou moins loin du contact avec sa propre détresse d'extimité. L'emmaillotera parfois de façon très bien trop - serrée. (De cela, et de ce qu'il est possible d'en faire à contre-pied, Alain Buffard parla dans son précédent opus, Good Boy, à travers une scène de superpositions de slips si folles qu'elles en venaient à empêcher tout mouvement; on comprend qu'il fallait danser!) Le Freud aussi qui, à la fin de sa vie, jette sur le papier cette note énigmatique, sur laquelle je m'étais déjà, ici même, l'année dernière, arrêtée: "Psychè est étendue. N'en sait rien.» ${ }^{13}$ Je garde pour l'heure en réserve l'usage que j'aurai à faire ici de cette note, et reviens à Lacan inventant lui aussi ce mot d'extimité, formé, on l'aura compris, à partir du terme d' «intimité»: formé à partir de ce terme non pour en être le pendant, mais plutôt pour sinon ruiner, du moins questionner sa pertinence. L'effet en tout cas de cette trouvaille lexi-

12 Michel Leiris, Biffures, Paris, Gallimard, 1982, p. 12.

13 Sigmund Freud, in Résultats, idées, problèmes, t. II, p. 288. 
cale sera de produire comme une généalogie de l' «intimité », et partant de dévoiler les enjeux qui couvent dans cette sphère en aucun cas préservée ou «libre de droits », si l'on peut dire. Enjeux qui concernent, j'y viendrai dans un instant, ce que j'appellerai les relations d'emprise. C'est donc en lien étroit avec ce que recouvre la notion d' «intimité», familière à tout un chacun - cela aussi dont parle Freud lorsqu'il parle de l'Unheimliche -, que Lacan fera jouer ce terme. Car ce doublet, extimitélintimité - dont Alain Buffard fera œuvre dans une opération qui sera quant à elle plutôt de disjonction, et j'aurai ultérieurement à revenir sur la leçon, pour le psychanalyste, d'une telle disjonction - éclaire d'un jour extraordinairement limpide l'énoncé de Lacan aux termes duquel «l'inconscient, c'est le discours de l'autre». Où l'on retrouve la question de la prescription, en ses enjeux les plus vifs et les plus violents, l' «intime» venant prescrire - au sens ici encore indissolublement double de ce terme, l'extime: cet innommé que Lacan osa nommer, et que Jacques Alain Miller, on verra comment, renvoie quant à lui dans l'innommable: nulle trace, dans l'édition officielle du Séminaire Vll, L'Éthique de la psychanalyse, de ce vocable, semble-t-il purement et simplement escamoté. Un signifiant que Miller a pourtant, d'une manière sur laquelle je viendrai dans un instant, et qui ne manque pas d'intérêt pour notre affaire, abondamment commenté, lui consacrant en 1986 son cours sur Lacan. À la place du très parlant néologisme imaginé par Lacan, nous trouverons «proximité ». Terme qui, s'il n'est certes pas absurde en l'occurrence, reconduit cependant non sans motifs le partage intérieur/extérieur que pulvérise le mot «extimité».

Si l'inconscient, soit ce qui, à son insu, meut le sujet, est « discours de l'autre », si ce qui est censément le plus «intime», le plus secrètement caché, dérobé au sujet lui-même, est ainsi tissé d'altérité première, et que cette altérité existe au sein du sujet comme discours, c'est-à-dire comme un ensemble signifiant actif, potentiellement source d'effets, que ce discours ait par ailleurs été ou non effectivement articulé, cela signifie que le «propre» de chacun est de toutes façons sous juridiction étrangère, au sens où nous en parlions plus haut, et n'existe tout simplement pas hors de cette constitution. Pareil «discours de l'autre», comme c'est plus ou moins le cas de tout discours, et plus encore de celui que l'enfant, encore infans, capte de l'adulte, n'est en aucun cas limpide. Mais il est porteur d'enjeux, d'amour notamment, et, si obscur, si ambigu soit-il - et peut-être à proportion de son obscurité -, il appelle comme une soumission à son sens supposé, sous peine de cette catastrophe qu'est la perte d'amour. Ainsi fonctionnera-t-il en premier lieu comme injonction, voire, dans ses plus sombres aspects, comme chantage pour le sujet, sommé, dans l'interprétation qu'il bricole de l'énigme de ce discours, ce discours qui partant le gouverne et l'identifie, d'être tel ou tel. Autrement dit, l'inconscient ainsi très freudiennement défini par Lacan, fonctionnera comme pure structure de lien. Un lien qui, en certains de ses points, plus ou moins nombreux, plus ou moins emmêlés, s'intensifiera en liens d'emprise, si l'emprise nous rapporte à l'autre sur un mode qui veut effectuer comme une soudure, mais aussi une suture, des désirs mutuels les uns sur les autres. Ici, par ce que noue, à la six-quatre-deux le plus souvent, l'interprétation, alors nécessairement univoque, de la teneur désirante dont seraient porteurs les mouvements perçus par un sujet comme émanant d'un autre, mouvements faits de mots, certes, mais aussi de gestes, de regards, voire de rien du tout. Interprétation qui, tordant le cou à l'énigme affolante dont sont porteurs ces mots, ces choses, et ces riens, les remplit 
jusqu'à ras bord d'un sens susceptible d'opérer ce que j'appellerais volontiers - et nous rejoignons l'affaire qui nous occupe - une réduction métaphorique. Réduction au sens où l'on parlerait de l'opération par laquelle on réduit une fracture; la fracture de l'ambiguïté qui menace de laisser seul au monde celui qui se sera trompé de sens. Terreur du malentendu. Mais l'envers de cet endroit de l'inconscient, de cet endroit qui exigerait que l'on ne se méprît point sur le sens unique, de cet endroit féroce - et l'on comprend là ce que veut exprimer Freud lorsqu'il indique que le Surmoi est pour une part essentielle inconscient -, impitoyable à l'encontre de tout contrevenant à l'orthodoxie ainsi constituée, orthodoxie impérieuse à raison de la dose d'arbitraire qui l'aura arrêtée, cet envers sera précisément, comme je le mentionnais plus haut, la puissance contenue dans la capacité de rêver: de faire foisonner la poéticité promise dans la donne métaphorique première. De cela, quel est l'enjeu exact? Les lignes qui vont suivre tenteront d'en poursuivre l'élucidation. Mais d'emblée je soutiendrai que cet enjeu est celui même qui détermine le pari, risqué, de l'exercice psychanalytique. Quant à la manière d'entrer dans ce pari, elle relève d'une position, sur laquelle je vais maintenant m'expliquer davantage, revenant, mais pour m'en démarquer, au commentaire que fait Jacques Alain Miller de Lacan, dont il dérobera par la suite à la vue le terme d'extimité.

Comment s'en sortir? C'est la question qui anime quiconque s'adresse à un psychnalyste. Cette question, c'est bien entendu celle de l'emprise, et derechef je soutiendrai que l'emprise est très précisément ce sur quoi - et avec quoi - travaille la psychanalyse, dont le dispositif spécifique, ne l'oublions pas, dégagea la question du transfert des processus à l'œuvre dans un autre dispositif, celui de la suggestion hypnotique. Dispositif quant à lui rentabilisant les conditions d'une relation d'emprise, conditions tributaires de la production d'une sorte d'enceinte à deux pôles que leur huis-clos rendra indissolubles. Point que Freud avait indiqué, comme en passant, dans un texte intitulé Traitement psychique, texte à la frontière du dispositif de la suggestion, au bord de l'invention de la psychanalyse: parlant du «rapport», terme qui désigne le lien de l'hypnotiseur et de l'hypnotisé, Freud écrit, jouant sur le mot Einreden, que l'hypnotiseur persuade/parle-dans: les traducteurs français ont écrit qu'il «insinue l'hypnose par la parole ${ }^{14}$; plus exactement, il s'agira d'amener l'autre «dans»: geste même de la fabrication d'un « dedans ». «Parler-dans »: intimer, et là aussi, on peut jouer sur le registre sémantique de ce terme, ce que du reste Jacques-Alain Miller ne manquera pas de faire, à sa très particulière façon. En tout cas, de la relation d'emprise, l'intériorité, et ses hauts murs, sera la structure même, et c'est en ce point précisément que l'«intime»sera injonction, calmante et mensongère, à oublier l'extime. Et nous voici tout près du labyrinthe: de sa généalogie pourrait-on dire. Ce que nous avons sans doute pressenti dès lors que s'est trouvée formulée la question: «comment s'en sortir». Il va donc nous falloir la prendre à bras le corps, cette question du labyrinthe, non sans avoir précisé au passage que «comment s'en sortir?» est aussi la question du cauchemar. Le cauchemar, cet enfermement sans recours autre que le réveil - et encore ! - dans une dimension de réalité - se situât-elle dans l'abandon du sommeil - qui n'autorise aucune échappatoire, aucun jeu, aucun

14 Sigmund Freud, Traitement psychique, in Résultats, idées, problèmes, t. I, p. 14. 
écart par rapport à ce qu'elle impose comme certitude. Autrement dit, dans l'état de cauchemar, ce qui est figuré adhère comme une ventouse à la conviction qu'il induit, aussi rend-il inconcevable qu'il puisse en être autrement que ce qu'il assène. Le cauchemar se donne comme implacablement réel, et partant radicalement univoque. En ce sens, il est l'anti-rêve par excellence, si le rêve, dans son fonctionnement même, source aussi du fonctionnement langagier, se déploie comme une sorte de feuilleté métaphorique, qui ne se laisse ni réduire ni épuiser. D'où le trouble qu'il fait naître, lié à ce que l'on pourrait désigner comme un léger, très léger, déficit de certitude, qui le décale de la conviction, intense, qu'il diffuse. Tout se passant comme si le rêve, lorsqu'il advient, certes prenait toute la place, mais que subsistait néanmoins toujours encore un peu d'espace vide, qu'il ne parvenait pas, dans son expansion indéfinie, à saturer complètement, voire même qu'il ne cessait de générer. Paradoxale situation, source parfois d'une certaine angoisse, fort différente cependant de la chappe dont nous écrase le cauchemar. Et l'expérience commune, qui témoigne de cette étrange état de fait, est que le plus souvent, du rêve, si transparent puisse-t-il nous apparaître, la compréhension que l'on croit avoir n'est jamais à proportion du sentiment, de la trace qu'il imprime en nous; nous nous échappons pour ainsi dire perpétuellement l'un à l'autre, comme en un jeu de cache-cache. Tandis que pour ce qui est du cauchemar, on est tout à fait certain, sans l'ombre d'un doute, qu'il met les points sur les «i » du tourment qu'il nous inflige: sans énigme ni aura, prison irrespirable, il nous enserre et nous broye, un point c'est tout.

Au point où nous en sommes, et si nous rassemblons, avant de faire un pas de plus, les différents brins qui forment notre écheveau, que pouvons-nous énoncer?

- En résumé, notre position de départ, aux termes de laquelle la psychanalyse est un art des passages, prend sens par rapport à la question du traitement des relations d'emprise entre les humains.

- L'inconscient est tout à la fois tissé de ces relations d'emprise - c'est sa définition comme «refoulé», voire «refoulé originaire»-, et possible espace de leur défaite: c'est sa puissance constitutive même, imaginative au sens de cette imagination inconsciente dont Freud parle comme capable d'initier la capacité onirique, et son art des détours. Détours susceptibles de jouer avec les corridors à la Piranèse qui nous retiennent et nous égarent, mais qui ne sont au bout du compte rien de plus que des marelles. Des marelles cependant auxquelles nous croirions dur comme fer; au Ciel qu'elles promettent comme à l'Enfer dont elles nous menacent si nous avons le malheur de toucher aux lignes de leur tracé.

- Ces relations d'emprise - et d'emprisonnement - constituent donc en labyrinthe - le labyrinthe de l'«intimité», soit de ce qui se construit comme l'enceinte d'un huis-clos - l'énigme de la relation structurellement et originairement métaphorique, c'est-à-dire fracturée depuis le début, d'un sujet au monde et à ce qu'il percevra comme «lui-même»; et l'on ferait sans doute mieux de dire, même si ça ressemble à du charabia, «lui-non-même»: extimité.

- La défaite de ces emprises a pour chemin ce qu'on pourrait appeler la puissance d'œuvre, soit la capacité à faire place à la poéticité qu'appelle, à l'infini, 
cette structure extime des singularités: poéticité, c'est-à-dire mouvement d'inscription, forcément paradoxal, bizarre mixte de douleur et de joie, pour faire venir au monde, sous une forme partageable y compris par soi(non)même, la présence toujours évanouissante de ce que c'est qu'être au monde. Espace dont il ne va pas de soi de supporter la dimension de solitude à quoi il expose. Une solitude d'autant plus menacée par les vents coulis qu'elle ne sera pas repli dans le cocon douillet du solipsme «intime».

- La métaphoricité à laquelle nous sommes, ce serait notre infirmité native, voués d'emblée, porte en elle deux destins opposés: sa réparation, par l'organisation du sens unique qui en prescrit l'usage. Sa rémunération, où l'on ne craindra pas de jouer à qui perd gagne. C'est la responsabilité, risquée, mais non sans une exigente prudence, de la conduite de la cure de psychanalyse.

- La psychanalyse est un dispositif que je conçois comme de rémunération de la métaphore. Mais elle peut aussi fonctionner au contraire comme tentative de réparation de celle-ci. Je soutiendrais qu'en cela, elle devient mensonge et abus de pouvoir, et se mue très exactement en cette technologie de normalisation que dénonçait Michel Foucault. Et cela a partie liée avec ce qu'on pourrait appeler l'injonction à l'intime. Technologie de normalisation, ce qui est tout autre chose que la mise au travail d'une normativité inventive, qui passe quant à elle par la reconquête d'un espace psychique ouvert à de l'inconnu.

Il me reste maintenant à examiner comment elle peut obéir à l'une ou l'autre logique que je viens de mentionner. Et pour cela à revenir au dispositif qu'elle inaugure: celui de la séance de psychanalyse. En reprenant appui sur l'énoncé freudien dont je tâche avec vous, depuis le départ de cette réflexion, de parcourir quelques arpents: «Psychè est étendue. N'en sait rien». Réflexion qui me paraît offrir une vision inédite, en tout cas non officiellement explicite chez Freud, même si elle est - métaphoriquement en certains textes - présente, de ce qu'est le refoulement, et partant de ce en quoi peut constituer sa levée, but de la cure. On peut soupçonner en tout cas d'ores et déjà ceci: à savoir que l'une des dimensions du refoulement serait précisément cette ignorance où se trouve la psychè de son étendue non enclose, c'est-à-dire d'une potentialité pour le sujet de déplacements autres que ceux qu'ordonnerait un espace clos - un labyrinthe -, et pour le coup d'invention des trajets de son existence et des figures de ses liens.

La séance de psychanalyse est un dispositif d'interlocution spécifique, dont le protocole obéit à une règle simple, dite règle fondamentale, qui se déploie en deux volets: du côté de l'analysant, énoncer une parole sous l'improbable régime de l'association libre; du côté de l'analyste, écouter cette parole dans l'élément d'une attention également flottante. C'est du reste cet aspect de la règle qui, pour pouvoir être réalisé autant que possible, appelle, comme seconde règle fondamentale de l'analyse, l'analyse de l'analyste. On comprendra très vite dans la suite de mon propos pourquoi, et que là encore, la règle «technique» est très directement éthique; il serait plus juste du reste de dire «politique», si l'enjeu de l'analyse est bien, au bout du compte, de rendre possible le questionnement des formes de vie, et non de nous plier par l'apprentissage d'une orthodoxie des labyrinthes. C'est-àdire: les liens d'emprise, nœuds coulants des ordres, hiérarchies, pouvoirs, normes, qui veulent nous faire marcher droit, faute de quoi nous chuterions dans 
le non-humain, ces liens dont l'indissolubilité censément radicale est la garantie qui saura verrouiller les structures de domination qui nous tiennent asservis, ces liens sont-ils susceptibles de se dénouer, sans que l'angoisse, ou la misère abandonnique ne s'emparent de nous?

C'est en ce point précisément, au carrefour de cette question, que nous retrouvons la problématique de la métaphoricité. Or la séance de psychanalyse, considérée aussi bien sous le point de vue du fonctionnement de la parole qu'elle requiert, que quant à l'étrange expérience psychique qu'elle induit - celle du transfert -, est un espace originairement, et de part en part, métaphorique. Un espace de décalage perpétuel du sens, au sein duquel justement, et parce qu'y sont mises en suspens les urgences de la vie, les métaphores sont sans cesse battues en brèche - et l'on pourrait utilement prendre ici à la lettre cette formule - en leur prétention à fournir adéquatement du sens et des images. On pourrait même soutenir qu'il est métaphorique au carré, en ceci qu'il redouble, démultiplie, et par là «fictionne», comme dirait Michel Foucault, réouvre par conséquent dans ses possibilités, l'écart initial dont nous sommes affectés: cette «extimité» que nous évoquions plus haut, source de l'invention du fameux proton pseudos. Invention dont il s'agira du coup d'exploiter les ressources. Et quant auxdites ressources, elles proviendront non de la teneur particulière de ce proton pseudos, qu'il s'agira au contraire de pouvoir décomposer, et laisser se recomposer autrement, hors de toute visée définitive, mais de l'espace même qu'inaugure son émergence. Un espace en tout cas dont il convient de remarquer ceci, qui intéresse notre problématique: il se verra configuré de telle sorte que puissent se disjoindre en lui, pour qui s'y aventure, conviction et certitude: rêve et cauchemar, œuvre et folie, cette folie fût-elle revêtue de l'uniforme gris, usé, poussiéreux, du conformisme le plus innocemment sûr de lui. Cette disjonction est une analyse, au sens le plus exact et le plus précis de ce terme.

Le détour ici par un texte de Nabokov, que je tire d'un roman intitulé La vraie vie de Sébastien Knight - et Sébastien Knight, le personnage, mort lorsque le livre commence, personnage dont son jeune frère cherche à écrire la biographie, est, tout comme Nabokov bien sûr, auteur «véritable» de la fiction dont Sébastien Knight et son frère sont les héros, l'auteur d'une œuvre -, ce détour me permettra de décrire plus précisément et d'expliciter ce que j'entends dans mon affirmation d'une métaphoricité fondamentale, et dirais-je refondatrice pour le sujet, de la séance de psychanalyse. Car l'expérience que déroule cette page, tout en parlant bien sûr de tout autre chose que d'une séance de psychanalyse, a pourtant comme une étrange ressemblance de famille, comme dirait Wittgenstein, avec l'expérience dont le dispositif analytique va produire les conditions. Voici ce texte:

...c'était comme si un voyageur se rendait compte que le pays sauvage sur lequel il pro-
mène ses regards n'est pas une réunion fortuite de phénomènes naturels, mais la page
d'un livre où ces montagnes, et ces forêts, et ces champs, et ces rivières, sont disposés de
manière à former une phrase cohérente; la voyelle d'un lac se soudant à la consonne
d'une pente; une route serpentante écrivant son message en ronde, aussi lisible que celle
de nos parents; des arbres conversant par signes, langage muet compréhensible pour
celui qui en a appris les gestes... Le voyageur épelle donc le paysage et le sens de celui-
ci se dévoile; il en va de même pour le motif compliqué de la vie humaine: l'on découvre
qu'il est monogrammatique, tout à fait clair maintenant pour l'œil qui désenchevêtre les
lettres entrelacées. Et le mot, la signification qui apparaît frappe de stupeur par sa sim-
plicité. [...] Et comme de toutes les choses la forme laissait transparaître la signification,
nombre d'idées et d'événements qui avaient paru d'une extrême importance étaient 
réduits, non à l'insignifiance, car rien à présent ne pouvait être insignifiant, mais à la taille même que d'autres idées et d'autres événements, à qui on déniait autrefois toute importance, atteignaient à présent. C'est ainsi que des géants brillants de notre esprit, tels que la science, l'art ou la religion, quittaient leur rang dans l'habituel système de classification et, se donnant la main, étaient mêlés et allègrement mis au même niveau. C'est ainsi qu'un noyau de cerise et son ombre minuscule sur le bois peint d'un banc vermoulu, ou un morceau de papier déchiré ou tout autre rien de ce genre, devenaient d'une taille prodigieuse. Remodelé et recomposé, l'univers livrait son sens à l'âme aussi naturellement que l'un et l'autre respiraient ${ }^{15}$.

Ajoutons que cette signification, pressentie dans l'œuvre de l'écrivain absent par le frère de Sébastien Knight, ne sera au bout du compte ni saisie ni fixée, mais, intensément présente, de façon diffuse, évanouissante autant que réelle, demeurera tapie dans l'éclat de la sensation qui ne parvient pas à en boucler la prégnance indubitable, claire, mais cependant, et à jamais, non assignable. Comme si plus la sensation, dans sa matérialité puissante, était vive, vraie, plus elle était aussi perception d'un incomblable écart entre les mots et les choses, pourtant étroitement entremêlés dans nos liens au monde et à nous-mêmes. Entre ces deux bords euxmêmes singulièrement mouvants, comme l'horizon qui s'éloigne à mesure qu'on s'en approche, et demeure ainsi toujours à bonne distance - cette distance fût-elle, d'un point $a$ et à un moment $t$ calculable -, s'étend le champ, indéfini, du mouvement métaphorique: de l'œuvre; de la vie humaine.

La séance de psychanalyse est un dispositif de décomposition du sens, et de sa recomposition kaléìdoscopique, tel que l'effet décrit dans le texte de Nabokov pourra y advenir: à savoir, indissolublement, la défaite des hiérarchies signifiantes qui voilent une vue exacte du tracé, sinueux, imprévisible, d'une existence humaine, et l'accès à la manière singulière, en partie aléatoire, dont se sont constitués pour un sujet, au sein du langage commun, si l'on peut dire sa grammaire et son lexique propres (le noyau de cerise, etc.). Or si cela peut advenir, c'est que la combinaison, clé de voûte de l'espace analytique, de l'attention également flottante de l'analyste, ce parfait étranger à l'histoire de celui qui parle sur le divan, et de la parole associative de l'analysant - et sans cette attention spécifique de l'écoute analytique, aucune associativité véritable de la parole ne pourra se déployer et prendre force -, cette combinaison donnera entièrement carrière au régime métaphorique, et ce sur plusieurs plans à la fois. On en dénombrera trois: l'un à l'œuvre dans la logique associative elle-même, puisque celle-ci est gouvernée par des déplacements issus de ressemblances, ces ressemblances fussent-elles improbables et fragmentaires. Ensuite, dans le déploiement de l'amour de transfert, c'est-à-dire du lien entre l'analysant et l'analyste, né de cette parole ouverte, il est en même temps question d'autre chose, d'autres et anciens liens d'amour, qui se mettront si l'on peut dire à flotter, et donc peut-être à pouvoir exister autrement que comme fixations d'emprises, dans l'élément de cet amour sans attachement qu'est l'amour analytique: sans attachement parce que indifférent à la vie particulière de chacun des protagonistes de cet étrange lien; et nous ne confondrons pas ici la particularité, dans laquelle toute vie est prise, mais où il est préférable qu'elle ne se trouve pas cloîtrée, avec la singularité, irréductible, même à coup de

15 Vladimir Nabokov, La vraie vie de Sébastien Knight, Paris, Gallimard, Coll. Folio, 1979, p. 268. 
particularités rassurantes, car toujours reconnaissables, identifiables. Car si le «particulier» est affaire de contenus, de ce qui fixe ces derniers et en détermine les contours, donc d'objets, un tel aspect n'intéresse nullement la question du «singulier», où il s'agira au contraire de percevoir des positions, des mouvements, des trajectoires, et donc des façons, toujours uniques, à l'infini de leurs métamorphoses, d'engendrer l'espace. Ainsi l'amour de transfert, pour en revenir à lui, sera-t-il un amour de toutes façons métaphorique de part en part, pas moins véritable pour cela, et l'un de ses effets, non le moindre, sera de redonner accès à la manière dont les premiers liens qu'il répète furent eux-mêmes des métaphores, voire des métaphores de métaphores: des façons pour le petit humain de donner du sens, par traduction-invention tâtonnante, à cette extimité dont nous parlions plus haut. Sauf que, et là gît l'oubli de la métaphoricité en son ouverture indéfinie et partant proliférante - appelons refoulement un tel oubli -, cette traduction pourra s'être mise à fonctionner comme unique version requise du lien: intimation, fabriquant l'intimité en ses figures particulières, justement, ainsi que le cortège des asservissements qu'elle perpètre. Nous serons là au cœur de ce qui fait l'efficience des relations d'emprise. C'est en cela que la psychanalyse, art des passages, comme nous le soutenions en commençant, a pour politique de desserrer l'étau desdites relations d'emprise, par la réouverture, non sans douleur certes, d'un espace de métaphoricité non clos. Ce qui ne signifie pas qu'il ne puisse lui arriver, cyniquement, effrontément, de piétiner cettte politique, source, on le conçoit sans peine, d'une rétivité certaine aux différents diktats qui veulent nous faire marcher droit, au profit d'une autre peut-être à bien des égards plus juteuse et moins propre à produire l'indocilité. Sans doute rencontrerons-nous là l'un des ses plus inquiétants périls, j'y viendrai dans un instant. Mais il me faut auparavant en venir au troisième aspect par lequel la psychanalyse est un espace originairement métaphorique, mais après-coup, réitérant ainsi, et renouvelant par là même, la dimension d'après-coup dont nous avons montré plus haut comment elle inaugurait l'extimité foncière au travail dans toute singularité. C'est que, bien sûr, une cure de psychanalyse, de par la temporalité de toute histoire subjective, vient nécessairement en un temps second dans une vie, second de toutes les manières; mais en même temps, le dispositif qu'elle inaugure, radicalement neuf, inexpérimenté à chaque fois, a priori dans son fonctionnement, c'est-à-dire offrant aux méandres d'une vie un espace inédit, décalé, à partir duquel pourront se redéployer les métaphores initiales qui auront dessiné ces tours et détours, ce dispositif manifeste au plus clair, et matérialise, la fécondité inépuisée, et pourrait-on dire, la puissance onirique de ce temps de l'après-coup. Car la parole en analyse, prise dans l'élément du transfert, zone intermédiaire entre le rêve en sa logique et la vie en sa nécessité, donnera lieu, de par le crédit qui lui est prêté - un crédit lié à la libre capacité de rêver de l'analyste-, à une réinvention métaphorique, au prix de la dissolution d'un trop plein de sens qui aura saturé, alourdi, et paralysé, la «labyrinterrorisant», l'existence d'un sujet ainsi ligoté aux métaphores par lesquelles il se sera, en quelque jour lointain, lié à ses semblables. Ceci à condition, il importe de le souligner, que soit toujours maintenu ouvert l'écart entre la promesse de sens et tout accomplissement définitif de celle-ci. C'est pourquoi l'interprétation analytique ne saurait être une herméneutique - retrouver le «vrai» sens supposé caché, mais une secousse capable d'initier de la création signifiante; ou encore, une façon de couper, comme sur un rosier, une fleur à l'intersection 
même de l'arbuste où d'autres boutons pourront repousser et s'épanouir; et ce n'est pas n'importe où, nul ne l'ignore. Cela suppose, on le comprend, que l'extension, sans bords fixes, du sens soit sans cesse réinvestie. Et que l'analyse, par conséquent, puisse être ce patient apprentissage par psychè qu'elle est en effet étendue, et non labyrinthe, en dépit des apparences. Extimité, pas nécessairement horrifiante, et non le «sale petit tas de secrets». Et que la règle de l'analyse; «dites ce qui vous vient à l'esprit », ne saurait en aucun cas, comme le soutient Jacques Alain Miller commentant, et soumettant à réduction normative (il faut, dit-il, «normaliser» les «paradoxes lacaniens » ${ }^{16}$ !!! ce qui ne laisse pas de faire frémir quant aux enjeux d'une telle normalisation) l'invention lacanienne du terme d'extimité, se concevoir comme injonction, «intimation», dit-il, à «faire savoir», accès donné à l'«intime», viol légitime en somme, ou procédure d'aveu, comme dirait alors, à juste titre, Michel Foucault. Conséquence peu surprenante de ce forçage de la notion d'extimité, qui ferait de cette dernière, incarnée en la figure toute puissante de l'analyste la clé du verrou, alors bien sûr absolument nécessaire, de la structure dite d'intimité (ou l'analyste en Barbe Bleue...): l'effacement de ce signifiant, on saisit bien maintenant pourquoi, dans l'édition légitime que le gendre publiera. Histoires de famille, bien sûr, dans la version qu'en dévoilent très souvent les affaires d'héritages. Histoires de famillles, c'est-à-dire en l'occurrence d'emprises validées en dominations supposées garantes du bon ordre des choses. Dominations qui tiennent pour autant qu'elles assigneront d'ailleurs, justement, une certaine place aux femmes conforme à un modèle du «féminin» reposant précisément sur cette construction de l'«intimité», d'un «dedans» d'autant plus propre à être objectivé, et aisément asservi qu'il sera protégé, surtout de cette «pensée du dehors » où se déroulerait, librement, la puissance d'œuvre. Il y aurait là, mais c'est un autre chapitre, beaucoup à dire sur le destin, dans notre civilisation qui «n'autorise même pas les femmes à être sorcières !», comme l'écrit dans un roman délicieux ${ }^{17}$ Sylvie Towsend Warner, des femmes artistes. No more comment.

Que psychè soit étendue, et que les tropismes qui vont engluer les modalités usuelles de la relation à la question du féminin, ou plutôt des femmes, ces étrangement pareilles aux hommes, nonobstant la différence anatomique de sexe qui distingue les un(e)s des autres, effectuent le repliement en labyrinthe de cette extension, et par là même sa méconnaissance, un texte de Freud nous le montre de très jolie manière. Un texte tiré de L'inquiétante étrangeté, article tout entier dédié à cette affaire de l'extime. Voici ce texte:

\footnotetext{
Un jour que je flânais, par un chaud après-midi d'été, dans les rues inconnues et désertes d'une petite ville italienne, je tombais par hasard dans une zone sur le caractère de laquelle je ne pus longtemps rester dans le doute. Aux fenêtres des petites maisons, on ne pouvait voir que des femmes fardées, et je me hâtais de quitter la ruelle au premier croisement. Mais après avoir erré pendant un moment sans guide, je me retrouvai soudain dans la même rue où je commençai à susciter quelque curiosité, et mon éloignement hâtif eut pout seul effet de m'y reconduire une troisième fois par un nouveau détour. Mais je fus saisi alors d'un sentiment que je ne peux que qualifier d'unheimlich $[\ldots]^{18}$.
}

16 Jacques-Alain Miller, Extimité, cours sur Le Séminaire livre VII, 1985-86 (Dactylographie).

17 Sylvia Townsend Warner, Laura Willowes, Paris, Gallimard, Coll. Folio.

18 Sigmund Freud, L'inquiétante étrangeté, in L'inquiétante étrangeté et autres essais, Paris, Gallimard, 1985, p. 238. 
Expérience analogue à d'autres, que Freud décrira également dans ce même article: se trouver égaré dans une forêt où l'on tourne en rond, chercher la porte dans une pièce noire et se cogner toujours au même meuble sans la trouver, ou tomber fortuitement sur son propre visage dans une glace, sans l'identifier immédiatement: voir son extimité, alors immédiatement refusée. Pour revenir au «labyrinthe» italien de Freud, nous dirons simplement, décalant en navigation la marche dans la ville, que sur les étendues marines, on pourrait tomber sur des sirènes, qui ne sont pas exactement des minotaures... En une fascinante condensation, ce texte qui vacille entre rêve et cauchemar nous donne l'exacte mesure de l'enjeu qui nous occupe, s'agissant des mouvements de la psychè: étendue versus labyrinthe. Il s'en faudrait de très peu en tout état de cause, pour que cette situation ne bascule dans l'angoisse, et c'est, tout comme chez Kafka, la respiration du burlesque, signe de non-adhérence à la menace d'enfermement, qui parvient à l'en sauver. Il apparaît d'autre part clairement, à l'occasion de la petite expérience que relate ce texte, à travers ce qu'elle vient en quelque sorte métaphoriser, qu'une certaine peur, assortie d'une façon d'identifier comme altérité ce qui fait peur, fabrique le labyrinthe. Le labyrinthe: structure de clivage, durci dans les murs en dédale, de l'extimité en deux entités ennemies. Ainsi existera, encagée, repoussée, solennellement sommée de demeurer tout ce qu'il y a de plus extérieure à soi, mais exerçant une irrépressible attraction, de l'altérité faite de l'altération dont sa peur aura purifié le héros. Altérité qui prend ici la figure des prostituées, dames qui n'ont, comme tout analyste attentif le sait bien sûr, aucun rapport avec ce qu'il peut faire, et sont par ailleurs l'un des avatars du monstre féminin. Mais en même temps, et par cette sorte de généalogie plutôt légère et drôle, si chargée soit-elle, il apparaît aussi, et c'est précisément le sentiment ambigü d'unheimlich qui le signale, qu'en aucun cas le labyrinthe n'est nécessaire. Davantage, qu'il n'existe pas. Seule existe la marche qui lui donne consistance. Mais les pas qu'elle dessine ne sont pas les seuls possibles: danser aussi, par exemple, est chose humaine, et façon d'engendrer l'espace, alors entretissé au mouvement lui-même, comme sa respiration. Cette danse pourra être une simple marche ${ }^{19}$, dès lors que son pas ne sera pas obnubilé, obturé, étouffé, par l'évitement. Où la danse se déploierait comme la puissance onirique de la marche. La version qu'Alain Buffard donne de l'extime, décidément, dans les différents plans qu'elle étale, peut ô! combien inspirer la méditation de notre pratique d'analystes; laissons à Jacques Alain Miller la passion de l'orthodoxie.

«Psychè est étendue. N'en sait rien», donc. Mais peut en apprendre quelque chose. Ce qui signifiera, tout comme de pouvoir parcourir la terre avec pour seule frontière la ligne d'horizon, cette ligne qui n'est pas un couloir, et que nos propres déplacements déplacent d'autant, ce qui signifiera donc, si on en revient à ce dont est fait le le lien humain, à savoir ce que Wittgenstein appellait les jeux de langage, en leur extension indéfinie - puisqu'il est toujours possible de créer un nouveau jeu -, que l'opération métaphorique, ce déplacement incessant du sens - le sens comme horizon, justement -, ne saurait se clore. Saturée, elle peut être meurtrière. Et disant cela, je pèse mes mots.

19 Voir entretien avec Alain Buffard, in «Vacarme», $N^{\circ} 7$, sur la marche comme «état absolu de la danse». 
Déplier le rêve, donc, qui replié, est labyrinthe. Déplier le rêve, dans cet analogon de son espace processuel incessamment métaphorisant qu'invente le dispositif de parole de la séance. Analogon en effet, puisque la logique onirique condensation, déplacement, par ressemblance - est précisément celle que réitère l'association libre. Dispositif dont on peut soutenir qu'il offre un lieu à une matérialisation de l'extime, un lieu sis au bord, actualisé, de l'existence de l'analysant. Ainsi peut-être quelqu'un pourra-t-il, singulièrement, trouver les voies pour que sa vie puisse prendre forme comme son rêve continué par d'autres moyens. Son rêve avec ses «prochains», comme écrivait Freud. Loin d'un quelconque solipsisme, mais au prix cependant d'une inévitable solitude.

«Je me déplace dans l'espace pour dilater le temps», écrivait sur l'écran de son ordinateur le personnage d'un film d'Élia Suleiman, intitulé Chronique d'une disparition. Énoncé qu'on pourrait imaginer réversible pour le sujet en analyse, lequel pourrait fort bien décrire son propre voyage en disant: «Je me déplace dans le temps (dans la mémoire enfouie des premiers liens, des premiers tâtonnements qui ourdirent la trame des premières métaphores) pour dilater l'espace.» L'espace pour respirer et vivre. Pour déplier son rêve et lui trouver son pas.

Paris. 